\title{
RESEARCH
}

\section{Computed tomography for dental implants: the influence of the gantry angle and mandibular positioning on the bone height and width}

\author{
JA Dantas ${ }^{*, 1}$, A Montebello Filho ${ }^{1}$ and PSF Campos ${ }^{2}$ \\ ${ }^{1}$ Department of Dental Radiology, Piracicaba Dentistry School, University of Campinas, Brazil; ${ }^{2}$ Department of Dental Radiology, \\ School of Dentistry, Federal University of Bahia, Brazil
}

\begin{abstract}
Objectives: To investigate the effect of deviation of mandibular positioning, by changing the gantry angle, on the measured height and width of dental implant sites in reformatted cross-sectional computed tomography (CT) scans.

Methods: CT images of ten human dry mandibles were made in three gantry positions to simulate changes in patient positioning: (1) parallel to the lower base of the mandible (standard); (2) with a gantry inclination of $+19^{\circ}$; and (3) with an inclination of $-19^{\circ}$. One examiner measured the bone height and width at selected sites in the images at three different times. Results were compared with a paired test in SAS 8.02.

Results: In relation to bone height, when the jaws were inclined to the inferior direction (gantry angle $+19^{\circ}$ ), there was no statistically significant difference for any region studied. There was a statistically significant difference for the incisor region when the jaws were inclined to the superior direction (gantry angle $-19^{\circ}$ ). With respect to the width of the bone rim, there was a statistically significant difference only for the region of the molars when the jaw was inclined to the inferior direction and for the region of the canine, when the inclination was to the superior direction.

Conclusions: Errors in mandibular positioning of $19^{\circ}$ produced image discrepancies with regard to bone height and width which were not excessive. Thus, examinations do not have to be repeated owing to variation of mandibular positioning because the differences were lower than $10 \%$ of the value found for the standard position.
\end{abstract}

Dentomaxillofacial Radiology (2005) 34, 9-15. doi: 10.1259/dmfr/57866813

Keywords: tomography; X-ray computed; dental implants; mandible

\section{Introduction}

Implants have been widely used in the replacement of lost dental elements, representing one of the major advances in terms of oral rehabilitation. However, in order to be successful, a rigorous evaluation of the quantity and quality of remaining bone and the accurate location of anatomical structures are necessary.

Computed tomography (CT) as well as other radiographic techniques provides a bidimensional representation of tridimensional structures; however, in CT, each section can be individually visualized, thus minimizing structural overlapping. Hence the information obtained

*Correspondence to: JA Dantas, Av. sete de setembro, 1942, ap. 501, Vitória, SSA/BA - Brazil, CEP: 40080001; E-mail: janadantas@yahoo.com.br Received 10 December 2003; revised 29 July 2004; accepted 26 August 2004 through CT can be more efficiently used as compared with that from conventional techniques.

In implantology, CT-guided surgery allows the visualization of internal bone morphology in three dimensions; therefore, the dental surgeon can plan precisely the insertion angle and implant length, thus enabling the anchorage of the apical portion on the cortical bone available. ${ }^{1}$

Despite its numerous advantages, studies have reported that measurement errors occurring in CT images for implants may be related to fault in positioning the patient's head, which cause cross-sectional images, obtained through multiplanar reformatting, not to be fully perpendicular to the lower border of the mandible. ${ }^{2}$

However, little is known about the actual effects of incorrectly positioning the patient during the acquisition of 
axial scanning in CT, particularly in relation to the effect of such variation in cross-sectional reformatting used to determine the height and width of the alveolar residual ridge needed for the placement of a dental implant. Therefore, this work aims to evaluate the influence of patient positioning on the achievement of axial slices for implant planning, observing the differences in the clinical measurements of bone height and width between standard mandibular position and both upper and lower mandibular position.

\section{Material and methods}

Ten human dry mandibles were provided by the Anatomy department of Piracicaba Dental School, after approval by the institution's Ethics Committee. Completely edentulous mandibles with entirely resorbed alveolar processes were selected for this study. They were randomly chosen with regard to their other anatomical characteristics.

The evaluated regions, which were bilaterally analysed, presented the following locations: incisor $(1 \mathrm{~cm}$ distal from the median sagittal plane), canine $(1 \mathrm{~cm}$ distal from the incisive region), premolar (to the level of the mental foramen) and molar ( $1 \mathrm{~cm}$ distal from the mental foramen), denominated I, C, PM and M, respectively (Figure 1). With the purpose to give a pattern to each one of the evaluated regions, eight radiopaque markers $(3 \mathrm{~mm}$ hollow metal spheres) were fixed on the occlusal surfaces of each mandible.

The images were acquired by a high resolution spiral CT equipment (CT HiSpeed Helicoidal DX/i; General Electric Co., Tokyo, Japan). The mandibles were maintained in a fixed positioning on a styrofoam surface, attached to the head holder of the CT equipment in order to simulate the patient's correct positioning in which the mandibular base was perpendicular to the horizontal plane (Figure 2).

Two millimetres thick axial tomographic sections were made with $1 \mathrm{~mm}$ intervals at $80 \mathrm{kV}$ and $60 \mathrm{~mA}$ and field of view $(\mathrm{FOV})$ of $15.8 \mathrm{~cm}$. On each mandible, the first axial scan was made parallel to the lower border. The images

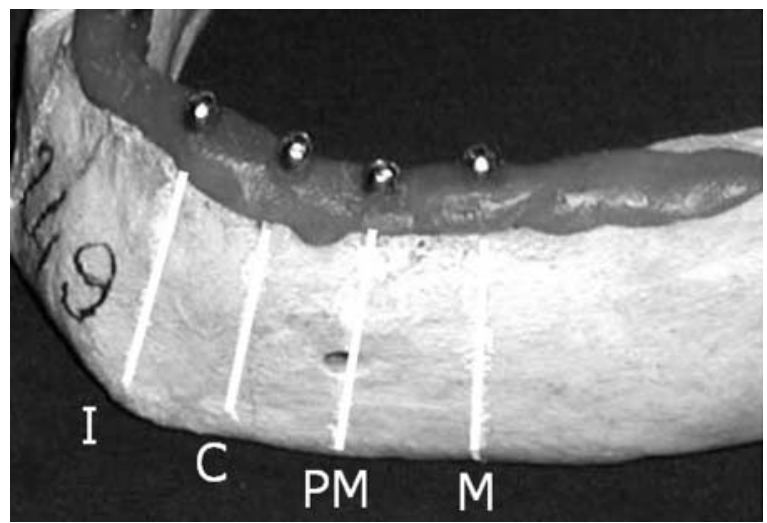

Figure 1 Evaluated regions: incisor $(\mathrm{I})$, canine $(\mathrm{C})$, premolar $(\mathrm{PM})$ and molar (M)

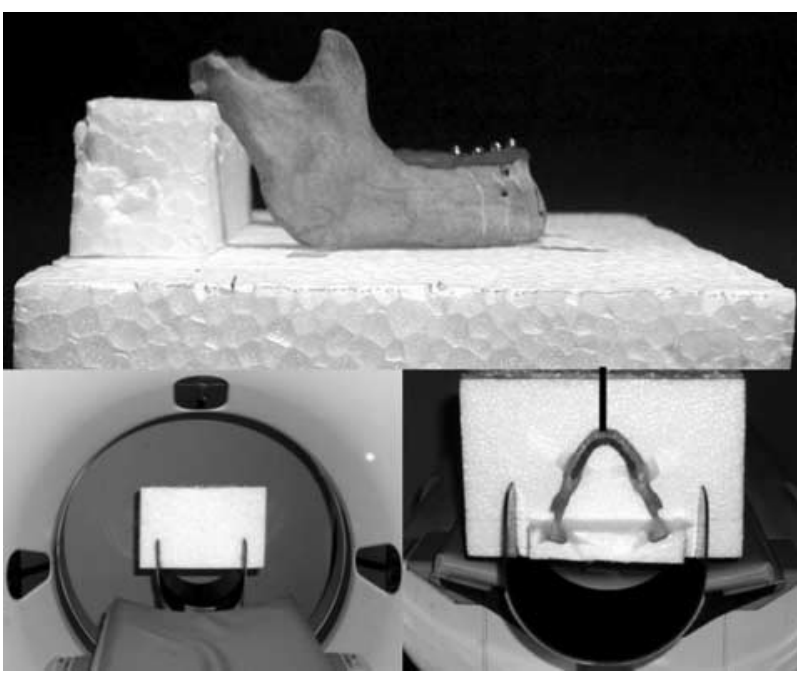

Figure 2 Diagram representing a jaw fixed to the positioner and such set adapted to the patient's headrest

obtained through this procedure were denominated as standard-position images (SP).

Next, to simulate the patient's altered positioning, which is a common clinical occurrence, new axial sections were performed with the mandible in the same fixed position but with a deviation of the gantry angle in two directions, $+19^{\circ}$ and $-19^{\circ}$, to simulate mandibular inclination. Based on the physical principle of relative movement, it is possible to determine that an object is moving, or not, depending on the chosen reference point, without having any effect on the obtained result. As all movement is relative, it is possible to consider either the mandible or the gantry objects in movement. For convenience in this study, the gantry was used as the reference point, perpendicular to horizontal plane, and the mandible was considered moving exactly in the same inclination used before, but to the opposite direction.

Using this principle, it was assumed that when the gantry was inclined to $+19^{\circ}$ it simulated a lower inclination of the mandible and a $-19^{\circ}$ gantry position simulated an upper inclination (Figure 3). Those were respectively denominated as image with lower mandible inclination (LP) and image with upper mandible inclination (UP).

After that, all the axial CT data were transferred to a workstation (Advantage Workstation 3.1 Ultra $10 \mathrm{sm}$; GE Medical Systems, USA), to generate panoramic and crosssectional reformatting images by the DentaScan software (General Electric Co., Wisconsin, USA). Those images were printed on a radiographic film by a chemical printer (969 Laser Imager; 3M Electrical products division plant, Minnesota, USA).

The same window amplitude was used for each mandible in the three different positions, so that the three tomograms of each would present an image with the same brightness and contrast standards.

Under ideal conditions of luminance and illuminance, the images were submitted to a single examiner who 


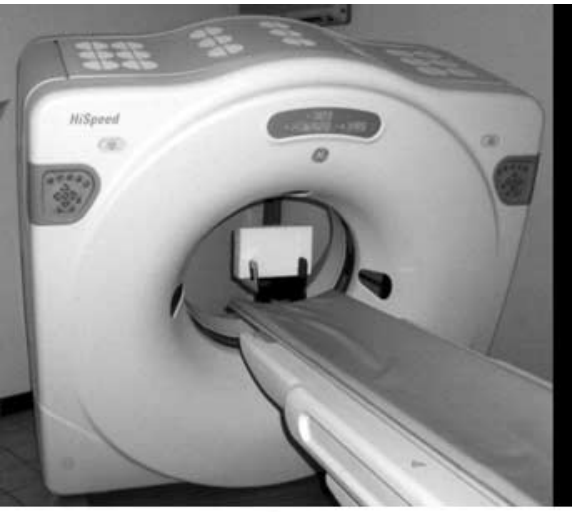

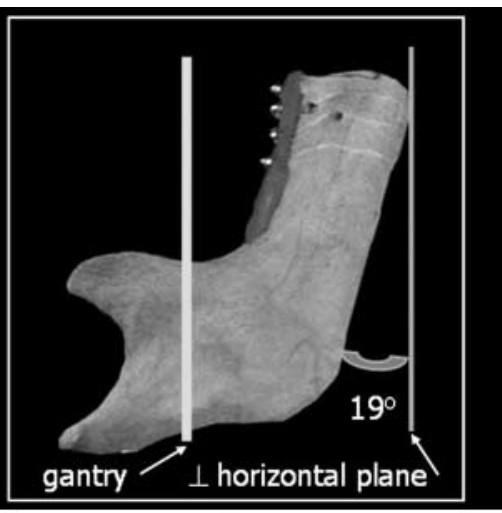

b

Figure 3 Acquisition of sections in the lower position (LP) of the jaw: (a) gantry position for sections acquisition; (b) diagram representing the transfer of the gantry inclination to the jaw

evaluated them at three different moments with a 2 weeks' interval between the examinations.

To obtain the measurements, cross-sectional images were used where the radiopaque markers appeared more defined in each of the selected areas. In order to determine clinical measurements, a specific anatomical structure was used as a reference point for each of the studied regions. The incisor and canine measurements were taken using the mandible's inner cortex as a reference, for the premolar area the limit was the upper border of the mental foramen and for the molar area the upper border of the mandible canal was chosen.

At the investigated region, a line was drawn tangent to the lowest point of the mandible. Starting from this point and perpendicular to this tangent, another line was obtained passing through the centre of the bone profile, equidistant from the buccal and lingual cortical plates, which was herein defined as bone profile line (BPL) (Figure 4). Next, with the help of a digital calliper and using this above-mentioned line (BPL) as a parallel reference, vertical measurements were taken starting from the crest of the mandible to the reference point selected for each studied region. Such measurements were denominated implant bone height (IBH). After that, horizontal measurements were taken, perpendicular to BPL, on the reference point used for the studied region, being denominated as bone edge width (BEW) (Figure 5).

The opening amplitude of the digital calliper corresponding to each measurement was determined by the

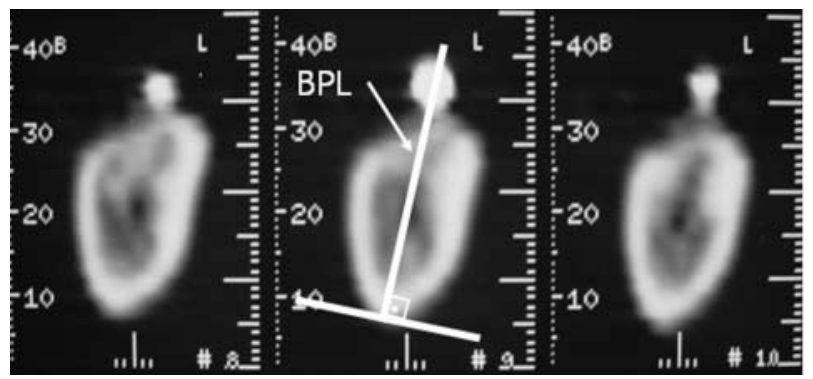

Figure 4 Determination of the bone profile line (BPL) millimetre scale of the tomographic film, thus preventing any type of image magnification that could interfere with the measurements obtained.

The values found were submitted to statistical analysis by using the software SAS 8.02 (Statistical Analysis Systems, Institute Inc., USA, 1999) and adopting an alpha significance level of $5 \%(\alpha=0.05)$. The means of the variables under study, height and width, in lower and upper position were compared with those in standard position separately in each of the evaluated regions, using paired tests.

\section{Results}

As all the $P$-values were greater than $5 \%$ among the bone height measurements between standard and lower positions, there were no statistical differences between the means in any of the investigated regions. However, the molar region approached the existence of a significant difference. In this way, it is possible to state that there was weak evidence $(P<0.10)$ of differences between the true means of these positions (Figure 6).

Also, in relation to the bone height, but now comparing the standard and the upper position of the mandible, the

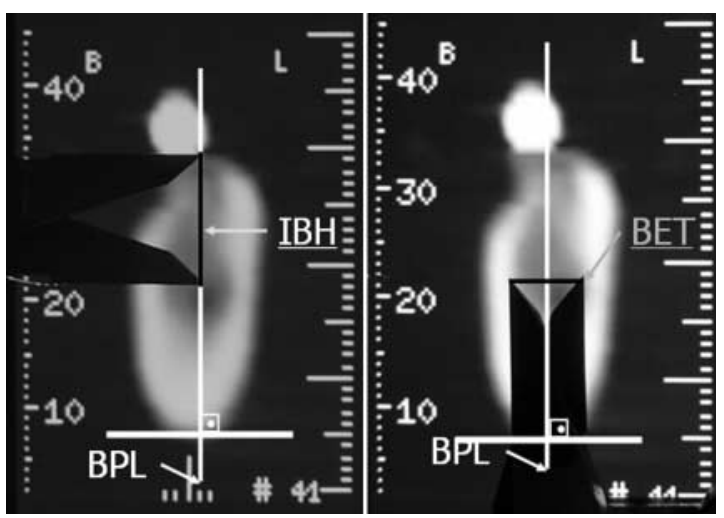

Figure 5 Determination of implant bone height (IBH) and bone edge width (BEW) measurements for the molar region 


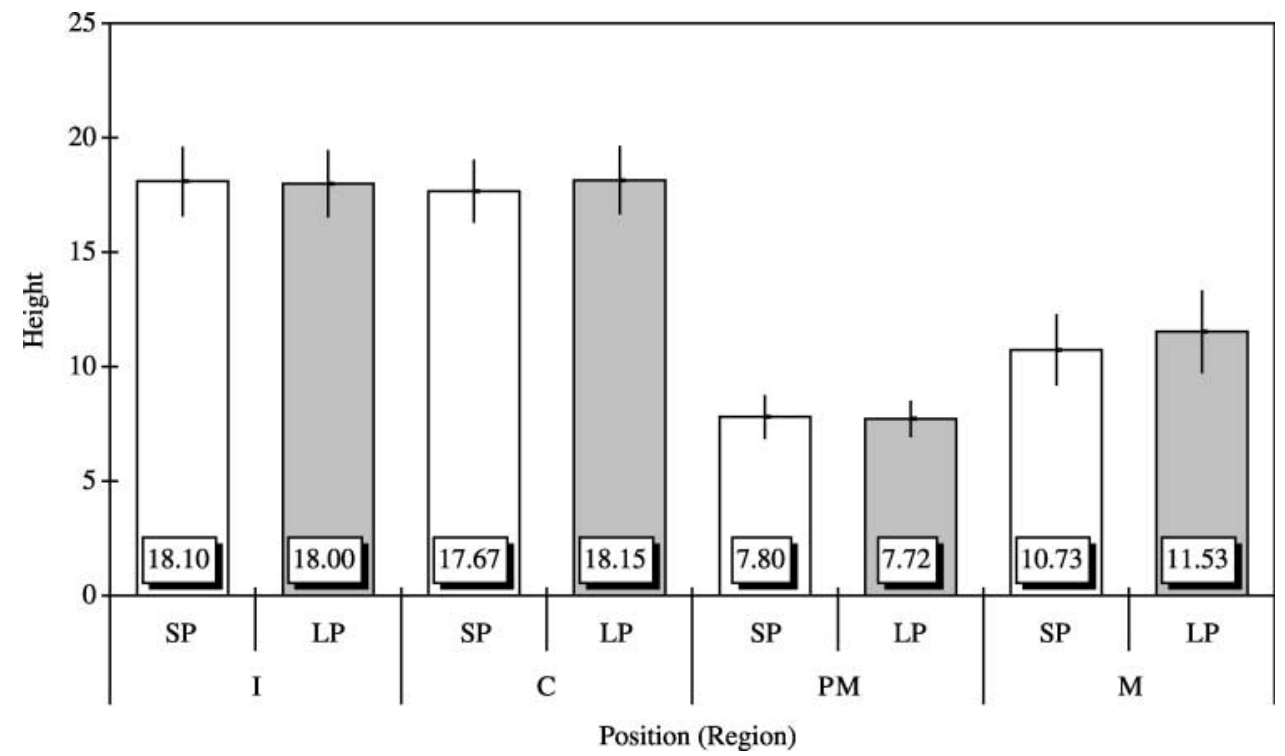

Figure 6 Comparison between the means and confidence intervals (95\%) of the variable height in standard position (SP) and lower position (LP), in the different regions under study

analysis of the test results for paired data showed strong evidence $(P<0.01)$ of statistically significant differences between the positions for the incisor region (Figure 7).

Concerning the bone width measurements in the standard and the lower positions, a higher mean for standard position in the molar region was observed during the analysis of test results. That difference was significant when compared by the Student's $t$-test with a significance level of $5 \%(\alpha=0.05)$ (Figure 8).

Finally, still regarding the bone edge width measurements between the standard and upper mandible's position, a strong evidence $(P<0.01)$ of differences between the means was found only in canine region. In all other regions, there was no evidence of differences between the true width measurements (Figure 9).

\section{Discussion}

The use of CT for planning implants has been intensively advocated over the last few decades, ${ }^{1,3}$ and its superiority in relation to conventional radiographic examinations ${ }^{2,4}$ as well as to conventional tomography is demonstrated in the literature.

In this experiment, a choice was made to select anatomical regions most frequently submitted to treatment

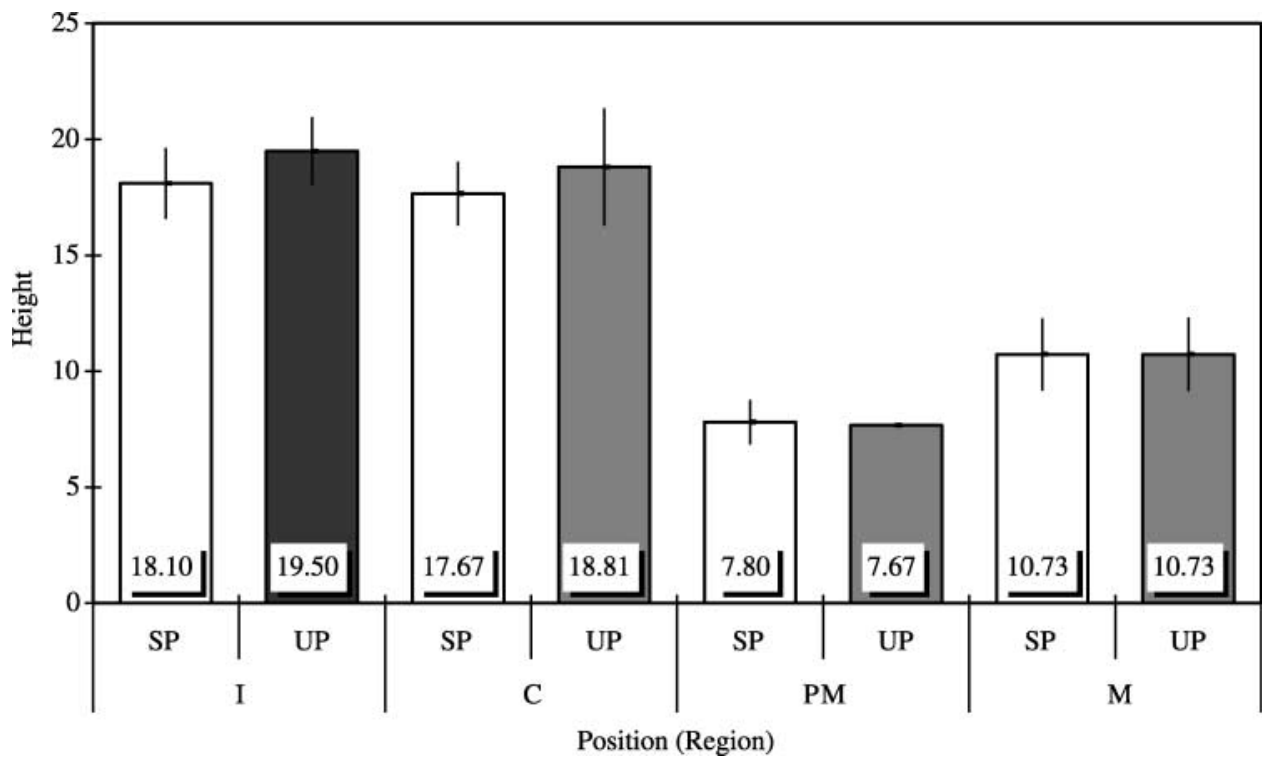

Figure 7 Comparison between the means and confidence intervals (95\%) of the variable height in standard position (SP) and upper position (UP), in the different regions under study 


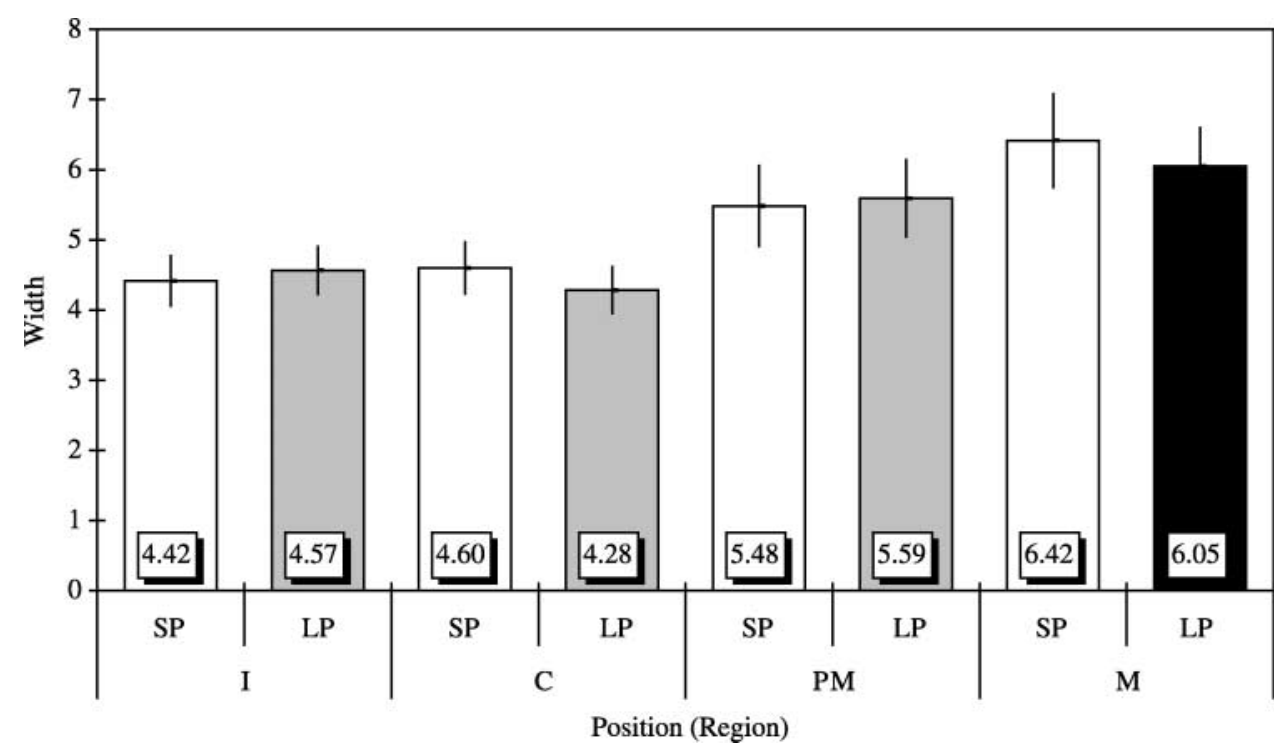

Figure 8 Comparison between the means and confidence intervals (95\%) of the variable width in standard position (SP) and lower position (LP), in the different regions under study

with implants, which were most likely to present surgical complications. In the case of the anterior mandible, in 2001, Akça and Iplikçioglu ${ }^{5}$ stated that such area was most frequently used for the placement of implants in completely edentulous patients at the same time that Tepper et $\mathrm{al}^{6}$ pointed out the importance of CT to evaluate that region. In 2002, Jacobs et $\mathrm{al}^{7}$ reaffirmed the validity of using cross-sectional CT in the evaluation of the anterior region in implant planning aiming at identifying the incisive canal in order to prevent potential sensory disturbances.

Concerning the premolar region, Cavalcanti et $\mathrm{al}^{8}$, in 1998 , and Bou Serhal et $\mathrm{al}^{2}$, in 2002 , conducted in vitro and in vivo experiments, respectively, and concluded that, by means of CT, accurate measurements could be performed in the mental foramen region, which is also considered to be of utmost importance for implant planning.

Nevertheless, no area has been the subject of so much discussion as the mandible's posterior region, which is where the mandibular canal is found. Its importance, its most frequent anatomical variations and particularly the accuracy of the measurements conducted in CT imaging for this specific area, have been extensively studied. ${ }^{3,4,9}$

As explained before, it is important to point out that although it was the gantry angle that was altered in this study, based on the physical principle of relative movement, all the measurements were done in terms of the

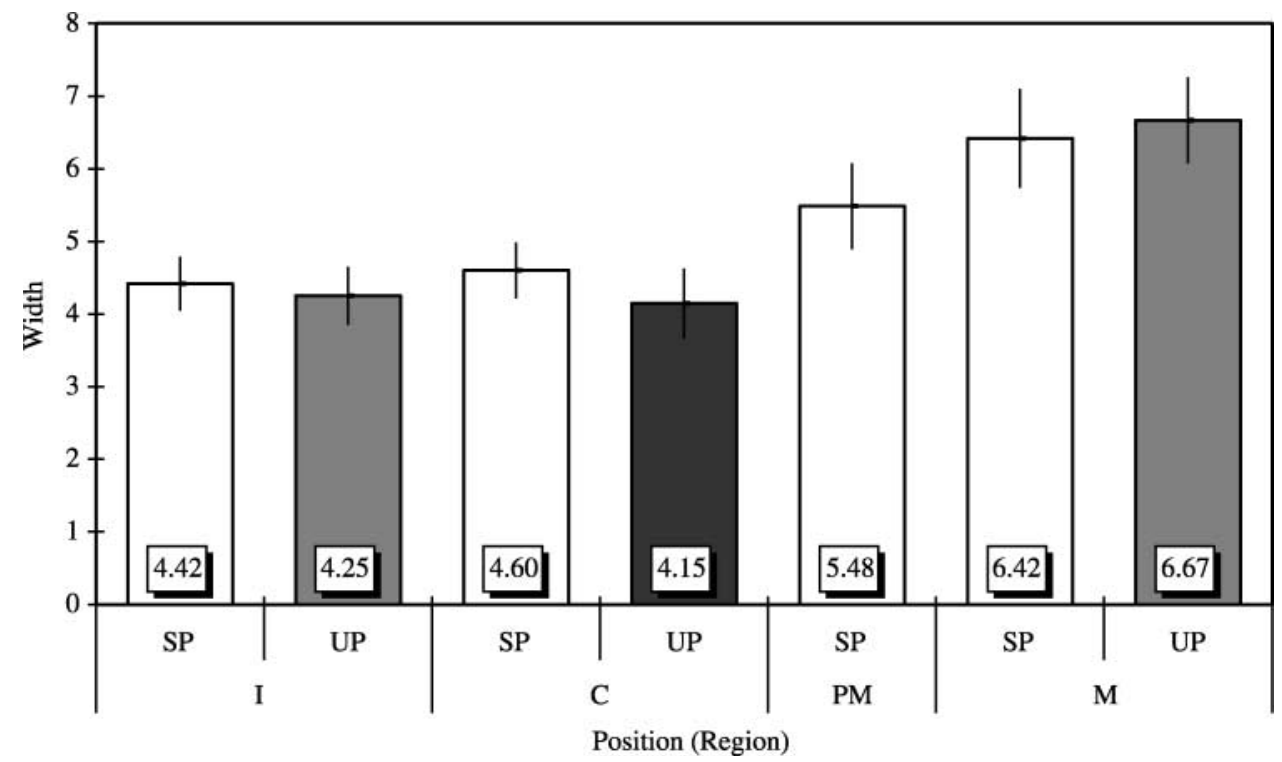

Figure 9 Comparison between the means and confidence intervals (95\%) of the variable width in standard position (SP) and upper position (UP), in the different regions under study 
mandible's inclination, which is a much more frequent clinical condition.

Concerning the measurement used, a choice was made to transfer the distance obtained by the digital calliper to the film's own scale, using the value found there. The selection of this procedure was based on studies which showed that the use of the film scale would eliminate the likely magnification found in the image printed on radiographic film. ${ }^{10,11}$

In addition, the standard position's images were used as a reference for comparison, as various studies, when comparing CT with physical measurements of the same region under study, showed that the size of measurements made on images with the correct positioning of the mandible were very similar to real ones, thus confirming the accuracy of such measurements. $3,9,12$

Based on the results found for the comparison of the variable height between the standard and the lower positions to those in the standard and the upper positions in each of the regions under study, it was possible to observe differences in the behaviours of incisor and molar areas. In the incisor region, when the mandible was inclined to the lower position, the differences found between the measurements were very small, whereas they were greater for the upper position, in addition to being statistically significant. It is believed that if the mandible is inclined while the sections are made, the crosssectional reformatting, instead of being perpendicular to the lower edge of the mandible, is obliquely reconstructed. As the measurement represents the distance between the alveolar bone crest and the mandible's internal cortical plate, a higher value is obviously found when a distance is diagonally measured. This was clearly demonstrated when the mandible's normal and upper positions were compared. On the other hand, it can be noticed that this large difference was not found when the lower position was evaluated, which can be explained by the parabolic shape of the mandibular bone. As the oblique section heads towards the mandibular symphysis, the bone's height difference was less obvious, in addition to the fact that the basilar cortical plate, which was the lower measurement limit, is thicker in the anterior region of the mandible, thus compensating for the diagonal measurement.

In relation to the molar region, when comparing the standard and the lower position, although the differences between the means were not statistically significant, they were much higher than those found for the upper position. In this case, the variation can be explained by the descending trajectory of the mandibular canal. In the upper position, thanks to such trajectory and since the canal was intercepted in a higher position, there was a compensation of the diagonal measurement. For the lower position, in addition to not being compensated, such diagonal measurement was exacerbated by the pathway next to the horizontal plane, as the mandibular canal heads anteriorly.

It is important to point out another factor concerning the situations where the measurements taken could not be included in the statistical analysis. In some cases, particularly at the premolar region of the upper position, it was found that when the mandible was inclined, in the cross-sectional image where the mental foramen was encountered, the radiopaque marker corresponding to this region was not found. And at the premolar marker, the mandibular canal was visible. This confirms that the differences found did not correspond to the distortions observed in the reformatted image, but rather to the performance of measurements in distinct sites.

However, concerning statistical analysis, whenever that situation took place, the measurements would have to be excluded from the sample, which negatively influenced the statistical analysis. Hence, a choice was made to remove the analysis of the variable width for the premolar region in the upper position.

On the other hand, although negatively influencing the statistical analysis, this situation confirmed the need and importance of using radiopaque markers in the regions of implant interest while acquiring CT images. Without them, it would be impossible to detect the occurrence of this situation, and by placing this fact in a clinical setting, it can be stated that their use ensured accuracy in the identification of the implant sites under study, which can be rather instructive in case of doubts concerning the fidelity of the regions presented in the image.

By comparing the results obtained in this work with data found in the literature about the influence of the mandibular positioning in CT imaging, it was observed that they were generally similar to those found by Kohavi et $\mathrm{al}^{12}$ in 1997 . By varying positioning, those authors found a difference mean of less than $3 \%$, which was considered to be a small margin of error and did not invalidate the use of CT. The authors suggested the adoption of a $10 \%$ safety margin in the measurements conducted on CT imaging for implants. When calculating the percentage values of the differences between the means observed in each of the regions herein studied, it was noticed that none of them were greater than that safety margin suggested by those authors. Therefore, the observed variation was very similar to that found by them (Table 1).

Furthermore, they observed that, by using a $0^{\circ}$ angle, there were no differences between the measurements obtained on the image and the physical measurements of the mandible. ${ }^{12}$ This shows that the images made when the mandible was correctly positioned, herein referred to as the standard position, presented a real size and could be used as a comparison pattern.

Another important study to be discussed is that by Choi et al, ${ }^{13}$ which was conducted in 2002 and aimed to evaluate the influence of variations in gantry angle on CT reformatted images. Those authors found significant

Table 1 Percentage values of the differences between the means of LP and UP in relation to SP

\begin{tabular}{lrrrr}
\hline & $I$ & $C$ & $P M$ & \multicolumn{1}{c}{$M$} \\
\hline Height SP $\times$ LP & $-0.6 \%$ & $2.7 \%$ & $-1.0 \%$ & $7.5 \%$ \\
Height SP × UP & $7.7 \%$ & $6.5 \%$ & $-1.7 \%$ & $0.0 \%$ \\
Width SP × LP & $3.4 \%$ & $-6.9 \%$ & $2.0 \%$ & $-5.7 \%$ \\
Width SP × UP & $-3.8 \%$ & $-9.8 \%$ & - & $3.9 \%$ \\
\hline
\end{tabular}

SP, standard position; LP, lower position; UP, upper position; I, incisor; C, canine; PM, premolar; M, molar 
differences between the measurements made on images obtained with a gantry angle different from $0^{\circ}$. Although the methodology applied in this work evaluated the mandibular positioning, a choice was made to keep it in a fixed position and to vary the gantry angle during the examination, thus attempting to obtain higher accuracy concerning the inclination angle used. Contrarily, in the study conducted by Choi et al, ${ }^{13}$ the testing samples followed the inclinations provided by the gantry. Therefore, image acquisition proceeded along the axis of the testing sample. Another relevant aspect is that the images were evaluated and measured in panoramic reformatting, different from the methodology applied in this work, which used the crosssectional images to do the measurements. Additionally, the authors pointed out the occurence of distortions on crosssectional images, a fact which is also contrary to the results obtained in this study that presents no image distortions, but rather an inaccurate indication of the position of such

\section{References}

1. Schwarz MS, Rothman SL, Chafetz N, Rhodes M. Computed tomography in dental implantation surgery. Dent Clin North Am 1989; 33: 555-597.

2. Bou Serhal C, Jacobs R, Flygare L, Quirynen M, Van Steenberghe D. Perioperative validation of localisation of the mental foramen. Dentomaxillofac Radiol 2002; 31: 39-43.

3. Yang J, Cavalcanti MGP, Ruprecht A, Vannier MW. 2-D and 3-D reconstructions of spiral computed tomography in localization of the inferior alveolar canal for dental implants. Oral Surg Oral Med Oral Pathol Oral Radiol Endod 1999; 87: 369-374.

4. Tal H, Moses O. A comparison of panoramic radiography with computed tomography in the planning of implant surgery. Dentomaxillofac Radiol 1991; 20: 40-42.

5. Akça K, Iplikçioglu H. Evaluation of the effect of the residual bone angulation on implant-supported fixed prostheses in mandibular posterior edentulism. Part I: Spiral computed tomography study. Implant Dent 2001; 10: 216-222.

6. Tepper G, Hofschneider UB, Gahleitner A, Ulm C. Computed tomographic diagnosis and localization of bone canals in the mandibular interforaminal region for prevention of bleeding complications during implant surgery. Int J Oral Maxillofac Implants 2001; 16: $68-72$. cross-sectional reformatting on the axial images, as stated by Besimo et al, ${ }^{14}$ in 2000.

Therefore, based on the analysis of the results found in this study, it was concluded that the differences herein observed did not represent a distortion of CT images, but rather an incorrect indication in the cross-sectional reformatting that, instead of being perpendicular, were oblique in relation to the mandibular base. Furthermore, the use of radiopaque markers helped to identify inadequately obtained scans, ensuring the accuracy of the regions under investigation. Finally, the percentage values of all the differences found were lower than $10 \%$ of the value for the standard position. In this way, when adopting a safety margin of $10 \%$, differences about $19^{\circ}$ in mandible positioning will not produce excessive discrepancies in the measurements of bone height and width that could cause the examination to be repeated, therefore exposing the patient to an unjustified dose of ionizing radiation.

7. Jacobs R, Mraiwa N, Van Steenberghe D, Gijbels F, Quirynen M Appearance, location, course, and morphology of the mandibular incisive canal: an assessment on spiral CT scan. Dentomaxillofac Radiol 2002; 31: 322-327.

8. Cavalcanti MGP, Yang J Ruprecht A, Vannier MW. Validation of spiral computed tomography for dental implants. Dentomaxillofac Radiol 1998; 27: 329-333.

9. Scher E. Risk management when operating in the posterior mandible. Implant Dent 2002; 11: 67-71.

10. Rothman SLG, Chaftez N, Rhodes ML, Schwartz MS. CT in the preoperative assessment of the mandible and maxilla for endosseous implant surgery. Radiology 1988; 168: 171-175.

11. Abrahams JJ. The role of diagnostic imaging in dental implantology. Radiol Clin North Am 1993; 31: 163-177.

12. Kohavi D, Bar-Ziv J, Marmary Y. Effect of axial plane deviation on cross-sectional height in reformatted computed tomography of the mandible. Dentomaxillofac Radiol 1997; 26: 189-191.

13. Choi S-C, Ann C-H, Choi H-M, Heo M-S, Lee S-S. Accuracy of reformatted $\mathrm{CT}$ image for measuring the pre-implant site: analysis of the image distortion related to the gantry angle change. Dentomaxillofac Radiol 2002; 31: 273-277.

14. Besimo CE, Lambrecht JT, Guindy JS. Accuracy of implant treatment planning utilizing template-guided reformatted computed tomography. Dentomaxillofac Radiol 2000; 29: 46-51. 